\title{
A tripla carga de agravos e os desafios para o Sistema Único de Saúde
}

Os processos de transição demográfica e epidemiológica colocaram as doenças crônicas não transmissíveis (DCNT) como principal causa de morbimortalidade no Brasil. Esse cenário levou o Ministério da Saúde a criar um plano de ações para enfrentamento dessas doenças, com medidas voltadas, sobretudo, para a vigilância e o controle de seus principais fatores de risco, cuja implantação se torna fundamental para a melhoria da qualidade de vida da população.

Por outro lado, embora as DCNT sejam responsáveis pela maior parcela da carga de doenças no Brasil, chama atenção a persistência de algumas doenças transmissíveis e as causas externas na composição do perfil de saúde do país. Os acidentes de trânsito e a violência interpessoal, além das infecções respiratórias e o HIV/AIDS, ainda contribuem com relevante parcela da carga de agravos na população brasileira. Ademais, o aperfeiçoamento dos programas de controle de doenças transmitidas por vetores, como dengue, chikungunya e zika, continuam exigindo esforços e são necessários para conter a expansão dessas doenças ${ }^{1,2}$.

Mais recentemente, a pandemia da COVID-19, causada pelo novo coronavírus, se constitui como um desafio sanitário mundial, exigindo resposta rápida e coordenada dos governos e sistemas de saúde, frente a uma doença de alta transmissibilidade em um mundo globalizado. A tomada de decisões pautada em evidências científicas robustas se faz necessária, havendo ainda um enorme caminho a ser percorrido. Desenvolvimento de vacinas, ensaios clínicos, ampliação da capacidade diagnóstica, estruturação de sistemas de vigilância e reorganização dos serviços de saúde são alguns exemplos desses desafios. No Brasil, medidas de distanciamento social têm conseguido adiar a explosão do número de casos e possibilitam uma melhor preparação dos serviços de saúde frente à pandemia, mas medidas para minimizar os danos econômicos, sociais e psicológicos, sobretudo das populações mais vulneráveis, se tornam fundamentais ${ }^{3}$.

Em pouco mais de 30 anos de existência, a expansão do Sistema Único de Saúde (SUS) contribuiu para reduzir a carga das doenças e agravos na população brasileira e reduzir as iniquidades regionais, mas ainda persistem desigualdades no acesso aos serviços e às ações de promoção e prevenção ${ }^{2}$. Nesse momento de enorme desafio para o controle da pandemia da COVID-19, aliado ao reconhecimento da relevante carga que outras doenças e agravos exercem na população brasileira, devemos lutar pela consolidação do SUS, como única estratégia para garantir o acesso universal e de qualidade à saúde no país. Além disso, deve-se ressaltar a importância de um sistema de ciência e tecnologia robusto e capaz de responder de forma rápida e eficiente aos diferentes agravos que acometem as populações, o que vem sendo ameaçado pelos crescentes cortes no financiamento para pesquisa no Brasil.

Neste número, a Revista Ciência \& Saúde Coletiva reúne artigos que demonstram o cenário da tripla carga de agravos para os sistemas de saúde, ressaltando a importância da produção de conhecimento para a adoção de medidas mais efetivas visando ao controle desses problemas. Os estudos aqui apresentados estão em consonância com a agenda de pesquisas estratégicas para o SUS, contribuindo para o fortalecimento desse sistema.

Sérgio Viana Peixoto (https://orcid.org/0000-0001-9431-2280) 1,2

${ }^{1}$ Instituto René Rachou, Fiocruz. Belo Horizonte MG Brasil.

${ }^{2}$ Escola de Enfermagem, UFMG. Belo Horizonte MG Brasil.

\section{Referências}

1. GBD 2016 Brazil Collaborators. Burden of disease in Brazil, 1990-2016: a systematic subnational analysis for the Global Burden of Disease Study 2016. Lancet 2018; 392(10149):760-775.

2. Souza LEPF, Paim JS, Teixeira CF, Bahia L, Guimarães R, Almeida-Filho N, Machado CV, Campos GW, Silva GA. Os desafios atuais da luta pelo direito universal à saúde no Brasil. Cien Saude Colet 2019; 24(8):2783-2792.

3. Werneck GL, Carvalho MS. A pandemia de COVID-19 no Brasil: crônica de uma crise sanitária anunciada. Cad Saude Publica 2020; 36(5):e00068820. 